\title{
The Effect of Eight Months of Twice-Weekly Low or Higher Intensity Whole Body Vibration on Risk Factors for Postmenopausal Hip Fracture
}

\section{Authors:}

Belinda R. Beck, BHMS(Ed), MS, PhD, Tracey L. Norling, BHS(HM)

\begin{abstract}
Affiliations:
School of Physiotherapy and Exercise Science, Gold Coast campus, Griffith University, QLD 4222, Australia
\end{abstract}

\section{Correspondence:}

Belinda R. Beck, BHMS(Ed), MS, PhD

School of Physiotherapy and Exercise Science

Gold Coast campus, Griffith University

QLD 4222, Australia

Phone: +61 (0)7 55528793

Fax: +61 (0)7 55528674

E-mail: b.beck@griffith.edu.au;

http://www.griffith.edu.au/health/school-physiotherapy-exercise-science/staff/dr-belinda-beck

\section{Disclosures:}

Financial disclosure statements have been obtained, and no conflicts of interest have been reported by the authors or by any individuals in control of the content of this article.

Funding was provided by a Griffith University Research Grant. There are no conflicts of interest related to the research or the manuscript. The authors did not derive any financial benefit from the work. Findings were presented as a poster at the Australian and New Zealand Bone and Mineral Society Annual Scientific Meeting, Sydney, March 2009 and the abstract printed in the conference proceedings. 


\title{
The Effect of Eight Months of Twice-Weekly Low or Higher Intensity Whole Body Vibration on Risk Factors for Postmenopausal Hip Fracture
}

\begin{abstract}
Objective: Whole-body vibration (WBV) is a potential therapy for age-related loss of musculoskeletal competence. Vibration has improved bone in animal models but human evidence is limited. Relative efficacy of low versus high intensity WBV is also unknown. Our goal was to observe the effect of brief low (LoWBV) and higher intensity WBV (HiWBV) on risk factors for hip fracture in postmenopausal women.
\end{abstract}

Design: We utilized an 8-month randomized controlled trial design to examine the influence of twiceweekly LoWBV (15mins, 30Hz, 0.3g) or HiWBV (2x3mins, 12.5Hz, 1g) on anthropometrics, bone (whole body, hip, spine, forearm, heel), muscle (wall squat, chair rise), and balance (tandem walk, single leg stance). Physical activity, daily calcium and compliance were recorded. Effects were examined by repeated measures ANCOVA, controlling for age, height, weight, calcium, physical activity, compliance, and baseline values.

Results: 47 women (71.5 \pm 9.0 years) completed the trial. There were no between-group differences in any measure at 8 months, but within-group effects were evident. Controls lost bone at the trochanter ($6 \%, \mathrm{p}=0.03)$ and lumbar spine $(-6.6 \%, \mathrm{p}=0.02)$ while WBV groups did not. WBV subjects improved wall squat (up to $120 \%, \mathrm{p}=0.004$ ) and chair rise performance (up to $10.5 \%, \mathrm{p}=0.05$ ).

Conclusions: 8 months of twice-weekly WBV may reduce bone loss at the hip and spine and improve lower extremity muscle function. These changes may translate to a decreased risk of falls and hip fracture.

Key Words: Bone, Muscle Function, Balance, Vibration, Hip Fracture 


\section{INTRODUCTION}

Despite quantum improvements in drug efficacy in recent decades, the impact of existing therapeutic options for osteoporosis remains modest. While pharmaceutical interventions have been shown to improve bone mass and decrease the incidence of fragility fracture, those improvements are not observed in all patients, some individuals suffer unacceptable side-effects, and compliance is poor ${ }^{1,2}$. Perhaps even more significantly, the vast majority of hip fractures occur as a direct result of a fall. Medications that improve bone mass do not generally influence neuromuscular performance ${ }^{3}$ and thus offer no protection from falls. To reduce falls, lower extremity muscle strength and balance must be addressed $^{4}$.

Although lifelong exercise is thought to optimize bone mass and neuromuscular function, for most, the intensity of exercise loading that can be tolerated in old age is unlikely to provide an adequate stimulus to notably improve bone. Moreover, despite the known systemic benefits of exercise, many people remain reluctant or unable to incorporate regular physical activity into their daily life ${ }^{5}$. The reluctance is particularly evident for those aged 65 and older $^{6}$, that is, those most at risk of hip fracture. Evidence is mounting, however, that passive exposure to low intensity mechanical loading may be osteogenic if applied at sufficiently high frequency; for example via whole body vibration $(\mathrm{WBV})^{7,8}$. It has been observed that roughly $80 \%$ of a $30 \mathrm{~Hz}$ vibration stimulus at the feet during standing can be transmitted to clinically-relevant bony sites (hip and spine) ${ }^{9}$. WBV may also improve muscle strength and balance, and thus has the potential to not only enhance bone health, but prevent falls ${ }^{10}$. Previous trials have predominantly examined the effect of WBV on most if not all days of the week. Considering the known challenges of patient compliance with most forms of treatment, determining the minimum effective dose of WBV is of critical therapeutic significance. The therapeutic dose of WBV with real life acceptability to populations at risk of hip fracture may be as few as 1-2 exposures per week.

The number of commercially available WBV devices has increased markedly in recent years. Where once the primary target market for vibration was the fitness industry, claims of clinical 
applications beyond the research evidence are now routinely advertised. The fact that substantial differences in stimulus intensity exist between the range of WBV devices is largely unrecognized or unacknowledged by the industry. In reality, evidence exists to suggest that cells can distinguish between different vibration frequencies and amplitudes ${ }^{11,12}$, such that the findings of studies examining effects of one WBV device cannot automatically be applied to another that operates at a different vibration intensity. It is of additional concern that many WBV devices operate at intensities beyond the safety limits described by the International Organization for Standardization (ISO 2631/3).

The aim of the current project was to determine and compare the effects of eight months twiceweekly, brief, low intensity WBV (0.3 g acceleration) versus relatively high intensity WBV (1 g acceleration) on risk factors for hip fracture (bone strength parameters, muscle function and balance) in independent living postmenopausal women. We hypothesized that: 1. eight months of twice-weekly whole body vibration would improve bone and muscle strength and balance; 2 . differences would be observed between effects of low and higher intensity WBV; and (3) treatment effects would be related to age, height, weight, initial bone mass, current physical activity, dietary calcium and compliance.

\section{METHODS}

\section{Ethical Approval}

All study activities were undertaken with the understanding and written consent of each subject, with the approval of the Griffith University Human Research Ethics Committee, and in accordance with the Code of Ethical Principles for Medical Research Involving Human Subjects of the World Medical Association (Declaration of Helsinki). 


\section{Study Design and Location}

An eight-month, randomized controlled intervention trial was conducted. We chose an 8 month study duration to optimise our ability to detect changes in bone, while minimising the risk of subject drop out that tends to increase with study duration. Supervised vibration sessions occurred twice-weekly at a local retirement village. Testing was completed in the Bone Densitometry Research Laboratory, Griffith University.

\section{Subject Recruiting and Sample Size}

Recruiting flyers were posted in many locations frequented by our target cohort including shopping centers and seniors clubs. Advertisements were placed in local newspapers and community newsletters. We also distributed flyers at retirement villages and ultimately based the study intervention at the retirement village from which we had the greatest recruiting response. Just under half of the participants resided in independent dwellings at the retirement village, with the remainder living in the wider community.

Women more than five years past menopause were enrolled to avoid the confounding effects of accelerated bone loss in the immediate postmenopausal years. Postmenopausal status was defined as the absence of menstruation for 12 months or more either after the age of 55 or after bilateral oophorectomy. Subjects were included if they were of sound general physical and cognitive health, fully ambulatory, and able to commit to twice-weekly participation for eight months. Subjects were excluded from the study if they had a metabolic bone disease, endocrine disorder or chronic renal pathology, had begun taking medications known to effect bone (including hormone therapy, bisphosphonates, parathyroid hormone, etc.) in the previous twelve months, or were recovering from lower limb fracture or other immobilizing injury.

In order to obtain sufficient statistical power to detect an effect in all dependent variables, we calculated the sample size required for the measure with the smallest expected effect size (i.e. bone 
mineral density - BMD). Studies of exercise interventions for bone in elderly cohorts commonly observe a rate of roughly $1 \%$ bone loss in control groups over twelve months ${ }^{13}$. To detect a $1 \%$ BMD difference with $0.8 \%$ standard deviation, at an alpha of 0.05 , for $80 \%$ power, eleven subjects were required in each

group. A standard deviation of $0.8 \%$ is modest, but commensurate with previous reports ${ }^{14}$. A minimum of four additional subjects were recruited into each group to accommodate potential attrition. True observed power is reported in the Results section as 1-ß after each statistically significant finding. Observed power averaged $70 \%$ for significant bone effects and $83 \%$ for muscle effects.

\section{Randomization}

The randomization scheme was generated using the first generator on the website Randomization.com (http://www.randomization.com, Dallal, 2007) for a three group trial. Subjects were assigned to the randomization scheme in the order of recruitment.

\section{Testing}

Age, height, weight, bone, muscle and balance parameters were measured at baseline and followup (eight months) as described below, by assessors blind to group allocation. Behavioral characteristics relevant to bone status, including dietary calcium and physical activity were recorded via questionnaire.

\section{Biometrics}

Subject height was measured to the nearest millimeter using a stadiometer (HART Sport \& Leisure, Australia). Weight was measured using digital scales (Soehnle Co., Switzerland) to the nearest 0.1 kilogram. Lean tissue and fat mass were obtained from whole body scans using an XR-36 Quickscan dual-energy x-ray absorptiometer (DXA) (Norland Medical Systems, Inc.) with host software, version 3.9.4, and scanner software version 2.0.0. 


\section{Bone Parameters}

The eight-month duration of the study was chosen as a time period over which changes in bone mass and size can be detected from DXA. DXA-derived bone area, mineral content (BMC) and mineral density (BMD) of the whole body (WB), non-dominant femoral neck (FN), trochanter (TR), lumbar spine (LS), and proximal forearm (FA) were examined. Femoral neck cross-sectional moment of inertia (CSMI) was calculated from DXA measures using the methods of Sievanen and colleagues ${ }^{15}$, to derive an approximation of bending strength.

Quantitative ultrasonometry (QUS) of the heel (QUS-2 Ultrasound Densitometer, Quidel Corporation, CA, USA) was used to determine broadband ultrasound attenuation (BUA) of the nondominant calcaneus. Calcaneal dominance was determined as the side with which a subject would kick a ball.

One investigator (BB) performed all bone measures. The coefficients of variation for BMD measures were as follows: $\mathrm{WB}-0.8 \%$, $\mathrm{LS}-1.2 \%$, FN $-1.4 \%$, proximal FA $-0.7 \%$.

\section{Muscle Function}

The wall squat and chair rise tests were used to examine muscle performance. These functional tests were selected for their simplicity and relevance to a daily task often associated with falling (lowering the body from standing). During the wall squat test, subjects stood comfortably on both feet with their back against a smooth wall. They then slid their back down the wall until a $90^{\circ}$ angle at the hip and knee was achieved. One foot was lifted off the ground, at which time the stop watch was started. The watch was stopped when subjects had to return the raised foot to the ground. Both limbs were tested and measures were taken in seconds. For the chair rise test ${ }^{16}$, the time to rise from and sit back down into a minimally padded straight-backed chair five times with arms folded, was recorded in seconds. One investigator (TN) performed all muscle measures. 


\section{Balance}

Static balance was measured using the single leg stance test (SLS). The length of time a subject could stand on their preferred limb without notable perturbation per the traditional protocol ${ }^{17}$ was measured in seconds. Dynamic balance was measured in seconds by the tandem walk test (walking heelto-toe on a straight line for six meters) ${ }^{16}$. One investigator (TN) performed all balance measures.

\section{Physical Activity}

A score of physical activity relevant to bone loading history was derived for each subject, from responses to a bone-specific physical activity questionnaire (BPAQ), previously described ${ }^{18}$ and available on-line (http://www.fithdysign.com/BPAQ).

\section{Calcium Consumption}

Dietary calcium was derived from responses to a calcium-focused customized Australian food questionnaire. Subjects were asked to indicate the type and amount of each food item consumed on average over a period of one day, one week, or one month. The average daily intake of dietary calcium was then determined using Foodworks (Version 5, Xyris Software Pty Ltd, Brisbane, Australia).

\section{Group Activities}

\section{Control Group}

Participants randomly allocated to the control group (Group 1) took part in baseline testing, and were then instructed to continue regular daily activities (and abstain from WBV) for a period of eight months before attending follow-up testing. All subjects were issued diaries in which they were asked to 
record falls, fractures, and changes in diet, medications and/or physical activity. Subjects were asked to avoid changing patterns of physical activity for the duration of the study period.

\section{Intervention Groups}

Participants randomly allocated to the intervention groups were tested, and then attended two sessions of supervised standing whole body vibration twice per week for eight months before follow-up testing. The low intensity whole body vibration (LoWBV) device (Juvent 1000 DMT, Somerset, NJ) is a flat, low set, relatively small platform providing a very mild vertical perturbation (Figure 1). The loading parameters of the LoWBV device were manufacturer settings of 0.3 g peak-to-peak acceleration at $30 \mathrm{~Hz}$ (root mean square power averaging $0.106 \mathrm{~m} . \mathrm{s}^{-2}$ ). Group 2 participants stood for fifteen minutes in full extension on the LoWBV device from the first session. The higher intensity whole body vibration (HiWBV) device (Galileo2000, Novotec Maschinen GmbH, Pforzheim, Germany) was larger, higher set, and comprised of a platform that oscillated around a sagittal axis at an amplitude of 0-14 mm, depending on foot position. With feet shoulder-width apart the stimulus amplitude was roughly $2 \mathrm{~mm}$. At an oscillation frequency of $12.5 \mathrm{~Hz}$, the stimulus acceleration was approximately $1 \mathrm{~g}$ (rms power averaging $0.52 \mathrm{~m} . \mathrm{s}^{-2}$ ). Group 3 participants stood with knees slightly bent for two bouts of three minutes separated by a one minute rest. The length of individual vibration bouts were progressively increased from one to three minutes over the initial two weeks. A single investigator (TN) supervised all intervention sessions, monitoring form and compliance.

The slight differences in intervention protocols reflect the fundamentally different theoretical premises from which high and low intensity WBV technologies were developed. The LoWBV device was developed from animal studies that indicated very low strains applied to bone around $30 \mathrm{~Hz}$ are most

osteogenic $^{7,8}$. HiWBV devices were originally developed to potentiate muscle training effects ${ }^{19}$. The idea that HiWBV will enhance bone is based on the notion that bone adapts as a function of muscle loading (or overloading), rather than a direct effect of vibration on the bone itself. It was important for 
reasons of practical translation to adopt WBV protocols around which each WBV technology was developed.

\section{Statistical Analyses}

Repeated measures ANCOVA with adjustments for multiple comparisons was used to examine between and within-group differences in intention to treat and per protocol analyses with a significance level of $\mathrm{p}<0.05$. Per protocol analysis excluded lost to follow-up and non-compliant subjects. Height, weight, age, baseline FN BMD, compliance, current physical activity and daily calcium consumption were controlled in analyses of bone parameters to account for their known influences on the skeleton. Age, compliance, and current physical activity were controlled in muscle and balance analyses.

Stepwise multiple regression analysis was employed to investigate the influence of group, compliance, age, percent change in weight, baseline values, current physical activity, percent change in lean mass, and daily calcium consumption on percent change of all bone parameters. Age, group, compliance, baseline values, and current physical activity were entered into the model for muscle and balance regression analyses.

All statistical analyses were performed using SPSS version 17.0 for Windows (SPSS, Chicago, IL).

\section{RESULTS}

\section{Subject Characteristics}

Forty-seven women (mean age, $71.5 \pm 9.0$ ) were eligible, consented and randomized to one of three groups; control (n=15), LoWBV (n=15) and HiWBV (n=17) (Figure 2.) Four subjects were lost to follow-up and one subject was excluded for failing to comply with the protocol. Of those, two were lost from the HiWBV group (1 dementia, 1 pelvic fracture), one from the LoWBV group (shingles), and one 
from the control group (declined follow-up testing). The subject excluded for non-compliance reported initiating and ceasing a number of bone medications during the course of the trial. Baseline group characteristics are presented in Table 1. The mean whole group femoral neck (FN) BMD T-score at baseline of $-2.2 \pm 1.1$ (range, $-4.18-0.6$ ) and Z-score of $0.17 \pm 1.1$ (range, $-1.55-2.94$ ) from the ITT analysis revealed the cohort exhibited bone mass in the normal range for age. As findings from the ITT and PP analyses were similar, we report per protocol data to reflect findings directly related to intervention exposure.

Groups did not differ significantly in age, anthropometric or behavioral characteristics at baseline, with the exception of weight which was greater in the LoWBV than HiWBV group $(68.4 \pm 10.3 \mathrm{~kg}$ versus $61.4 \pm 8.9 \mathrm{~kg}, \mathrm{p}<0.05)$.

\section{Compliance}

Treatment compliance was $92.1 \%$ for LoWBV participants and $91 \%$ for the HiWBV group. Examination of study diaries at follow up confirmed all subjects had complied with the request to maintain stable patterns of extraneous physical activity over the course of the trial.

\section{Falls}

Our study was not powered to detect a treatment effect on falls; however, we note that three subjects in the LoWBV group, one subject in the HiWBV group and six subjects in the control group fell once during the course of the trial. 


\section{Treatment Effects}

\section{Biometrics}

There were no between or within-group treatment effects on weight, height, BMI, percent fat or lean mass.

\section{Bone}

Repeated measures ANCOVA controlling for age, height, weight, daily calcium consumption, compliance and baseline FN BMD found no between-group differences in any outcome measure, however, a number of within-group effects were observed. As a within group analysis involves the examination of change in the same subjects from baseline to follow-up, the focus for this secondary analysis was on BMC rather than BMD as a more representative indicator of total bone mass at each site. Significant within-group effects indicated that controls lost trochanteric (TC) BMC (-6.0\%, $\mathrm{p}=0.03,1-$ $ß=0.60)$, spine (LS) BMC (-6.6\%, $p=0.02,1-\beta=0.65)$ and LS area $(-5.7 \%, p=0.0009,1-\beta=0.76)$ whereas vibration groups did not. LoWBV subjects increased $\mathrm{FN}$ area $(2.3 \%, \mathrm{p}=0.02,1-\beta=0.60)$. A significant increase in HiWBV subjects' calcaneal broadband ultrasound attenuation (BUA) (3.4\%, p=0.05, 1$ß=0.50$ ) was also observed, however power was low. Despite no observed loss at the hip or spine, the LoWBV group lost whole body (WB) BMC (2.1\%, $\mathrm{p}=0.03,1-\beta=0.70)$ and proximal forearm (FA) BMD (1.4\%, $p=0.005,1-ß=0.86)$ (Table 2, Figure 3).

Multiple regression analyses revealed only a modest ability of any independent variable to predict percent change in dependent bone variables. Percent change in lean mass accounted for $21 \%$ and $16 \%$ of the variance associated with percent change in WB BMC $(p=0.003)$ and WB BMD $(p=0.01)$ respectively; and group allocation accounted for $10 \%$ and $14 \%$ of the variance associated with percent change in LS BMC $(\mathrm{p}=0.04)$ and area $(\mathrm{p}=0.02)$, respectively. 


\section{Muscle}

No between-group differences were revealed for any muscle measure, however, within-group effects were again observed. Wall squat time improved significantly for the vibration groups but not the control group at the dominant leg (HiWBV, 70.9\%, p=0.02, 1-ß=0.80) and non-dominant leg (HiWBV, 119.5\%, $\mathrm{p}=0.004,1-ß=0.85$; LoWBV, 70.1\%, $\mathrm{p}=0.03,1-\S=0.65)$. Chair rise time also improved significantly in the vibration groups only (HiWBV, $-10.1 \%$, $\mathrm{p}<0.05,1-\S=0.55$; LoWBV, $-11.5 \%$, $\mathrm{p}=0.05$, $1-ß=0.50$ ), although power was low (Table 2, Figure 4).

No independent variable was significantly associated with percent change in wall squat or chair rise time in the regression analysis.

\section{Balance}

No whole group, between-group or within-group effects were evident for tandem walk or single leg stance time (Table 2, Figure 4). There were no significant predictors of single leg stance (SLS) time, but baseline tandem walk time predicted $11.6 \%$ of the variance in percent change in tandem walk $(p=0.03)$.

\section{DISCUSSION}

Our goal was to determine the effect of two sessions per week of brief, low intensity or higher intensity whole body vibration for eight months on risk factors for hip fracture in independent living postmenopausal women. To our knowledge, there has been no previous comparison made between vibration intensities in a randomized controlled trial, nor an examination of only twice-weekly exposure in the absence of an associated exercise program. Intention to treat (ITT) and per protocol (PP) analyses were performed to examine both the public health implication and actual treatment effects of our protocol. Findings from those analyses were essentially the same. No significant treatment effect of either form of 
WBV was observed for any variable. Despite the finding of no overall treatment effect, within group comparisons were statistically significant in a number of cases as indicated in Table 2. Although recognised as a less meaningful finding than a true between group treatment effect, within group effects can sometimes detect subtle findings that may be clinically significant, such as a reduction in loss, rather than a gain. This effect is particularly evident in Figure 3. Specifically, we found that control subjects lost significant trochanteric (TC) BMC (-6.0\%), spine BMC ($6.6 \%)$ and spine area $(-5.7 \%)$ while vibration groups did not. The changes exceed the coefficients of variation for each of those measures for our lab, which are $3.1 \%, 1.7 \%$ and $1.4 \%$, respectively indicating changes of these magnitudes are unlikely to be due to DXA measurement error. The vibration group subjects (particularly HiWBV) also exhibited significant improvement in muscle endurance (wall squat) while controls did not. An analysis comparing a combined vibration group (LoWBV + HiWBV) against controls produced similar findings for all variables.

Curiously, LoWBV subjects lost 2.1\% whole body BMC and 1.4\% proximal forearm BMD over the course of the eight month trial. It could be surmised from this bone loss observed at the proximal forearm (a cortical bone site not loaded by a standing vibration stimulus) and at the whole body (also predominantly cortical bone) that systemic bone loss unrelated to the vibration intervention was occurring more rapidly in the LoWBV than the HiWBV or control groups during the course of our study. Importantly, the LoWBV group did not lose significant bone at the hip and spine (indeed a slight increase occurred in femoral neck [FN] area), suggesting the vibration stimulus ameliorated the effects of systemic bone loss at sites most directly exposed to the stimulus.

The non-significant trend for an increase in TR BMC in the HiWBV group may reflect hip extensor loading of the trochanter during HiWBV to resist the cyclical passive flexion perturbation of the lower extremity during the up phase of the oscillating plate motion. As it is well recognized that reductions in bone size can result in loss of bone strength, maintenance of lumbar spine bone area in both WBV groups can be considered advantageous in comparison with the loss observed in the control group. 
A significant increase in femoral neck bone area in the LoWBV group contributed to a $7.4 \%$ positive trend ( $\mathrm{p}=0.09$ ) in FN cross sectional moment of inertia affirming the potential strength benefits of increased bone size even in the absence of improvement in BMC or BMD.

Observations at the calcaneus are consistent with the different stimuli to which subjects were exposed; that is, controls (no vibration) tended to lose $3.4 \%$ broadband ultrasound attenuation (BUA) (NS), LoWBV subjects (who experienced no ankle perturbation) exhibited essentially no BUA change, and HiWBV subjects increased BUA by 3.1\% $(\mathrm{p}=0.05)$. Although power in the BUA analysis was low, if the improvement was real, it is likely a reflection of the repetitive calf muscle loading of the calcaneus via the Achilles tendon as a consequence of the requirement to resist cyclical passive ankle dorsi flexion during oscillating HiWBV.

Notably, we did not observe a difference in effect between the two different WBV stimulus intensities. It is possible that the stimuli perceived at the level of the bones were not, in fact, different enough to engender a markedly different response. That is, although the HiWBV perturbation was considerably higher than the LoWBV at the level of the plate, with the introduction of a flexed joint stance, the stimulus was likely attenuated - potentially to the extent that the HiWBV stimulus perceived at the hip and spine was similar to that of the LoWBV. It is also possible that bone cells become desensitised at a certain threshold, such that more (i.e. higher WBV intensity) is not necessarily better.

Five somewhat comparable, randomized trials have examined LoWBV or HiWBV treatment effects on bone outcomes in postmenopausal cohorts. Findings from the lone LoWBV trial (same device as the current study; peak-to-peak acceleration of $0.3 \mathrm{~g}$ ) mirrored our observations. That is, there was no observed treatment effect of LoWBV, however, within group effects were apparent for the most compliant, light weight subjects. In that cohort, BMD losses at the hip and spine in controls were ameliorated by twelve months of $2 \times 10$ mins/day LoWBV ${ }^{20}$. Three of the four HiWBV trials are not entirely comparable to the current study as two included exercise during the vibration and one used walking as a control. Six months of 10 mins, $30 \mathrm{~Hz}, 5 \mathrm{~mm}$ amplitude vibration five times per week improved FN and LS BMD in postmenopausal women with osteoporosis ${ }^{21}$. Although not clear from the 
description of the data analysis it appears these differences were changes within group rather than treatment effects. Six months of static and dynamic knee extensor exercises for up to thirty minutes in addition to 35-40 $\mathrm{Hz}$ and $2.5 \mathrm{~mm}$ amplitude vibration (peak acceleration of up to $5 \mathrm{~g}$ ) improved hip BMD in comparison with resistance training alone or control ${ }^{22}$. Eight months of 6 x 1 min vibration, 3 x week at $12.6 \mathrm{~Hz}$ and $3 \mathrm{~mm}$ amplitude (in 60 degrees of knee flexion) improved FN (but not spine) BMD and balance in comparison with walking ${ }^{23}$. Eighteen months of twice weekly 25-35 Hz, 1.7 mm amplitude vibration superimposed on 15 minutes of dynamic leg strength training did not effect greater changes in bone mass than the exercise training alone ${ }^{24}$. A systematic review of the evidence for effects of vibration on muscle strength concluded that there is moderate to strong evidence that long-term vibration exercise improves lower extremity muscle performance in older cohorts ${ }^{10}$, which our results tend to support. A large well-controlled twelve month trial found HiWBV + fitness training was associated with reduced sway in response to stance perturbation in healthy older adults (mean age 66), but was otherwise comparable with fitness training alone ${ }^{25}$. Other reports indicate that HiWBV, with and without exercise, has variously improved balance and stability in nursing home residents ${ }^{26}$ and elderly community dwellers 22. While positive trends exist in our data, particularly for the HiWBV group who exhibited a tendency to improve single leg stance time by nearly 30\% (NS), we did not observe any significant balance effects. In light of the very brief nature of the intervention sessions and the small number of weekly exposures, a maintenance effect for some bone sites was somewhat unexpected. Few differences were apparent between low and higher intensity vibration maintenance effects on bone, however, HiWBV appeared to improve static muscle endurance (wall squat) to a somewhat greater extent than LoWBV. Effects were largely uninfluenced by age, height, weight, initial bone mass, current physical activity, dietary calcium or compliance. While our between-group differences were not significance, our withingroup observations suggest that there may be subtle fracture risk benefits to be gained from even very modest engagement in WBV for postmenopausal women. The reduction in loss of bone at loaded sites, combined with improved muscle function in squatting activities may reduce both risk of falling and risk of fracture should a fall occur. A tendency for a greater numbers of falls in the control group than either 
vibration group provides preliminary (but underpowered) support for a falls protective effect. Increased exposure to WBV may confer more substantial benefits and warrants further examination.

\section{Limitations}

The contribution of bone geometry to bone strength is well known. Bone geometry is best evaluated using three-dimensional technology, such as magnetic resonance imaging (MRI) or computed tomography (CT). As logistics prevented the latter measures in the current trial, we calculated an index of bone geometry, cross sectional moment of inertia (CSMI), from DXA measures using the methods developed and validated by Sievanen and colleagues ${ }^{15}$. We recognize this method limits our ability to directly detect an influence of the intervention on bone geometry. Bone marker data would also have assisted us to observe the effect of vibration on bone remodeling. Furthermore, it is acknowledged that non-equivalent WBV stimuli were applied in the LoWBV and HiWBV groups. As previously described, the justification for the approach was the desire to examine real-life manufacturers’ recommended protocols and reflect the different theoretical mechanisms of action, namely direct versus indirect (muscular) stimulation of bone. It is also noteworthy that despite adjusting for multiple comparisons in the analyses, subject numbers limited the statistical power of some comparisons. Large standard deviations created a risk of beta error, that is, a potential inability to detect a significant difference.

\section{$<$ INSERT TABLE 3 ABOUT HERE $>$}

As current therapeutic drug efficacy for osteoporotic fracture is limited by side effects, poor compliance and a lack of neuromuscular efficacy, there is a strong imperative to identify alternative preventative options. Twice-weekly treatment of either fifteen minutes of LoWBV or six minutes of HiWBV is a minor treatment impost, as evidenced by the excellent study compliance by our subjects.

It is important to re-emphasize that marked variation in stimulus intensities exist between the large range of WBV devices that exist in the commercial market. This dissimilarity limits the universal applicability of data collected from one WBV device to any other. In many cases, stimulus intensity of a 
device is not readily apparent. For this reason, efficacy and safety of the vast majority of WBV devices remain largely unknown. No side effects from either form of WBV were reported in our trial, however, one subject in the HiWBV group (FN T-score, -3.5) experienced a week of pain followed by a low trauma pelvic fracture four weeks after initiating the intervention. As the subject had sustained a low trauma pelvic fracture in the year prior to enrolling in the study her physician concluded the second fracture was not directly related to the intervention. It is unknown if the HiWBV stimulus contributed to the progression of the injury.

In conclusion, eight months of brief, twice-weekly whole body vibration, for either fifteen minutes at $0.3 \mathrm{~g}$ vertical acceleration $(30 \mathrm{~Hz})$, or six minutes at $1 \mathrm{~g}$ alternating limb acceleration $(12.5 \mathrm{~Hz})$ did not elicit detectable treatment effects in postmenopausal bone, muscle or balance measures.

However, within groups, WBV was associated with maintenance of bone at some clinically-relevant sites (proximal femur and lumbar spine), and improvements in lower extremity muscle function.

\section{ACKNOWLEDGMENTS}

The authors thank the staff and residents at Earle Haven Retirement Village (Gold Coast, QLD, Australia) and local community-dwelling subjects, for their enthusiastic engagement with our trial. 


\section{REFERENCES}

1. Carr AJ, Thompson PW, Cooper C: Factors associated with adherence and persistence to bisphosphonate therapy in osteoporosis: a cross-sectional survey. Osteoporos Int 2006; 17:(11): 1638-1644.

2. Cooper C, Jakob F, Chinn C, et al: Fracture incidence and changes in quality of life in women with an inadequate clinical outcome from osteoporosis therapy: the Observational Study of Severe Osteoporosis (OSSO). Osteoporos Int 2008; 19:(4): 493-501.

3. Bemben D, Langdon D: Relationship between estrogen use and musculoskeletal function in postmenopausal women. Maturitas 2002; 2: 119-127.

4. Wolfson L, Judge J, Whipple R, King M: Strength is a major factor in balance, gait, and the occurrence of falls. J Gerontol A Biol Sci Med Sci 1995; 50 Spec No: 64-67.

5. $\quad$ Gold Coast Physical Activity Survey. Gold Coast Population Health Unit, Queensland Health 2006: 1-55.

6. Participation in Sports and Physical Recreation, Australia, 2005-06. Australian Bureau of Statistics 2007: $1-11$.

7. Hsieh YF, Turner CH: Effects of loading frequency on mechanically induced bone formation. $J$ Bone Miner Res 2001; 16:(5): 918-924.

8. Rubin CT, McLeod KJ: Promotion of bony ingrowth by frequency-specific, low-amplitude mechanical strain. Clin Orthop 1994:(298): 165-174.

9. $\quad$ Rubin C, Pope M, Fritton JC, Magnusson M, Hansson T, McLeod K: Transmissibility of 15-hertz to 35hertz vibrations to the human hip and lumbar spine: determining the physiologic feasibility of delivering low-level anabolic mechanical stimuli to skeletal regions at greatest risk of fracture because of osteoporosis. Spine 2003; 28:(23): 2621-2627.

10. Rehn B, Lidstrom J, Skoglund J, Lindstrom B: Effects on leg muscular performance from whole-body vibration exercise: a systematic review. Scand J Med Sci Sports 2007; 17:(1): 2-11.

11. Judex S, Lei X, Han D, Rubin C: Low-magnitude mechanical signals that stimulate bone formation in the ovariectomized rat are dependent on the applied frequency but not on the strain magnitude. J Biomech 2007; 40:(6): 1333-1339.

12. Christiansen BA, Silva MJ: The effect of varying magnitudes of whole-body vibration on several skeletal sites in mice. Ann Biomed Eng 2006; 34:(7): 1149-1156.

13. Bravo G, Gauthier P, Roy PM, Payette H, Gaulin P: A weight-bearing, water-based exercise program for osteopenic women: its impact on bone, functional fitness, and well-being. Arch Phys Med Rehabil 1997; 78:(12): 1375-1380.

14. Beck BR, Kent K, Holloway L, Marcus R: Novel, High Frequency, Low Strain, Mechanical Loading for Premenopausal Women with Low Bone Mass: Early Findings Journal of Bone and Mineral Metabolism 2006; 24:(6).

15. Sievanen H, Kannus P, Nieminen V, Heinonen A, Oja P, Vuori I: Estimation of various mechanical characteristics of human bones using dual energy $\mathrm{x}$-ray absorptiometry: Methodology and precision. Bone 1996; 18:(1): 17S-27S.

16. Morris R, Harwood RH, Baker R, Sahota O, Armstrong S, Masud T: A comparison of different balance tests in the prediction of falls in older women with vertebral fractures: a cohort study. Age Ageing 2007; 36:(1): 78-83.

17. Vellas BJ, Wayne SJ, Romero L, Baumgartner RN, Rubenstein LZ, Garry PJ: One-leg balance is an important predictor of injurious falls in older persons. J Am Geriatr Soc 1997; 45:(6): 735-738.

18. Weeks BK, Beck BR: The BPAQ: a bone-specific physical activity assessment instrument. Osteoporos Int 2008; 19:(11): 1567-1577.

19. Roelants M, Verschueren SM, Delecluse C, Levin O, Stijnen V: Whole-body-vibration-induced increase in leg muscle activity during different squat exercises. Journal of strength and conditioning research / National Strength \& Conditioning Association 2006; 20:(1): 124-129.

20. Rubin C, Recker R, Cullen D, Ryaby J, McCabe J, McLeod K: Prevention of postmenopausal bone loss by a low-magnitude, high-frequency mechanical stimuli: a clinical trial assessing compliance, efficacy, and safety. J Bone Miner Res 2004; 19:(3): 343-351.

21. Ruan XY, Jin FY, Liu YL, Peng ZL, Sun YG: Effects of vibration therapy on bone mineral density in postmenopausal women with osteoporosis. Chin Med J (Engl) 2008; 121:(13): 1155-1158. 
22. Verschueren S, Roelents M, Delecluse C, Swinnen S, Vanderschueren D, Boonen S: Effect of 6-Month Whole Body Vibration Training on Hip Density, Muscle Strength, and Postural Control in Postmenopausal Women: A Randomized Controlled Pilot Study. J Bone Miner Res. 2004; 19:(3): 352-359.

23. Gusi N, Raimundo A, Leal A: Low-frequency vibratory exercise reduces the risk of bone fracture more than walking: a randomized controlled trial. BMC musculoskeletal disorders 2006; 7: 92.

24. Stengel Sv, Kemmler W, Engelke K, Kalender W: Effects of whole body vibration on bone mineral density and falls: results of the randomized controlled ELVIS study with postmenopausal women. Osteoporosis International 2010; DOI 10.1007/s00198-010-1212-4.

25. Bogaerts A, Delecluse C, Claessens AL, Coudyzer W, Boonen S, Verschueren SM: Impact of whole-body vibration training versus fitness training on muscle strength and muscle mass in older men: a 1-year randomized controlled trial. J Gerontol A Biol Sci Med Sci 2007; 62:(6): 630-635.

26. Bruyere O, Wuidart MA, Di Palma E, et al: Controlled whole body vibration to decrease fall risk and improve health-related quality of life of nursing home residents. Arch Phys Med Rehabil 2005; 86:(2): 303307. 


\section{FIGURE LEGENDS}

Figure 1: Whole body vibration devices in use during a study intervention session. HiWBV -higher intensity whole body vibration device; LoWBV - low intensity whole body vibration device

Figure 2: Subject enrolment and flow

Figure 3: Eight month within-group percent change (mean $\pm \mathrm{SE}$ ) in selected bone parameters following twice-weekly whole body vibration; per protocol analysis $(\mathrm{n}=42)$. HiWBV - higher intensity whole body vibration; LoWBV - low intensity whole body vibration, WB - whole body; TR - trochanter; LS lumbar spine; PFA - proximal forearm; BMC - bone mineral content; BMD - bone mineral density; CSMI - cross sectional moment of inertia; BUA - broadband ultrasound attenuation. Significantly different from baseline measure: ${ }^{\mathrm{a}} \mathrm{p}=0.03,{ }^{\mathrm{b}} \mathrm{p}=0.02,{ }^{\mathrm{c}} \mathrm{p}=0.009,{ }^{\mathrm{d}} \mathrm{p}=0.005,{ }^{\mathrm{e}} \mathrm{p}=0.05$

Figure 4: Eight month within group percent change (mean $\pm \mathrm{SE}$ ) in muscle and balance measures following brief, twice-weekly whole body vibration; per protocol analysis $(\mathrm{n}=42)$. WS - wall squat test; DOM - dominant leg; NDOM - non-dominant leg; SLS - single leg stance. Significantly different from baseline measure: ${ }^{\mathrm{a}} \mathrm{p}<0.02,{ }^{\mathrm{b}} \mathrm{p}<0.004,{ }^{\mathrm{c}} \mathrm{p}<0.03,{ }^{\mathrm{d}} \mathrm{p}<0.05$ 


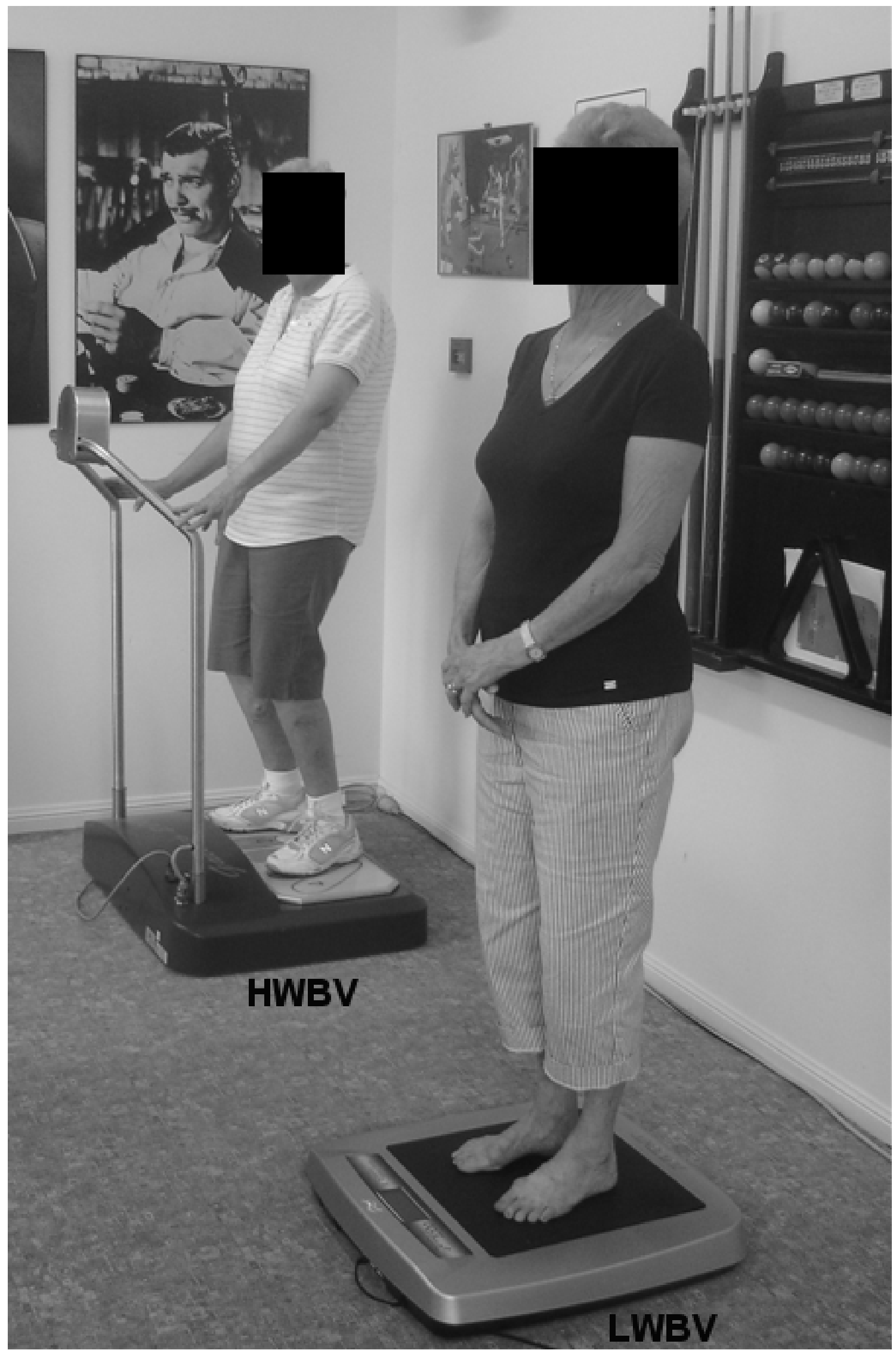




\section{CONSORT diagram of subject flow}

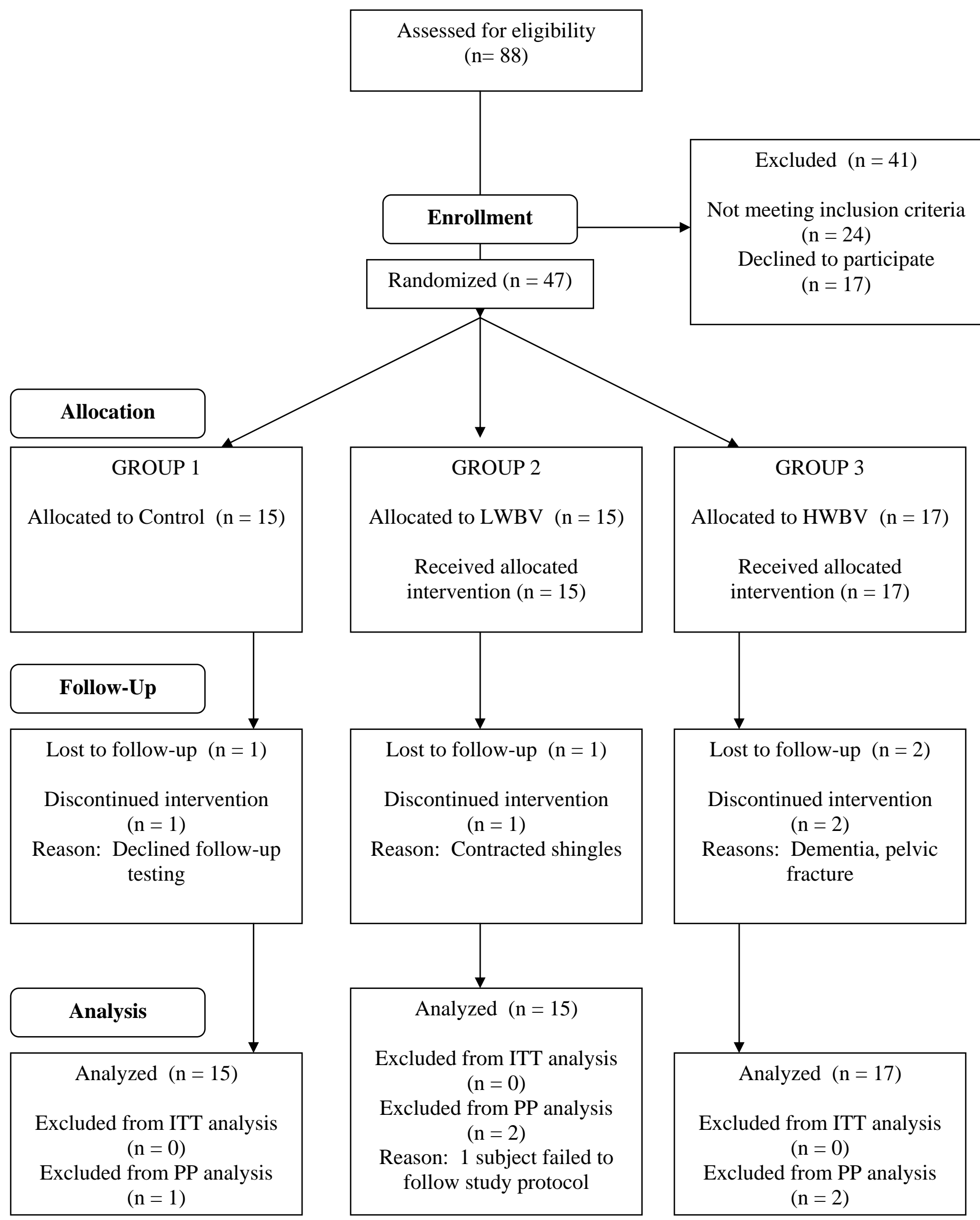




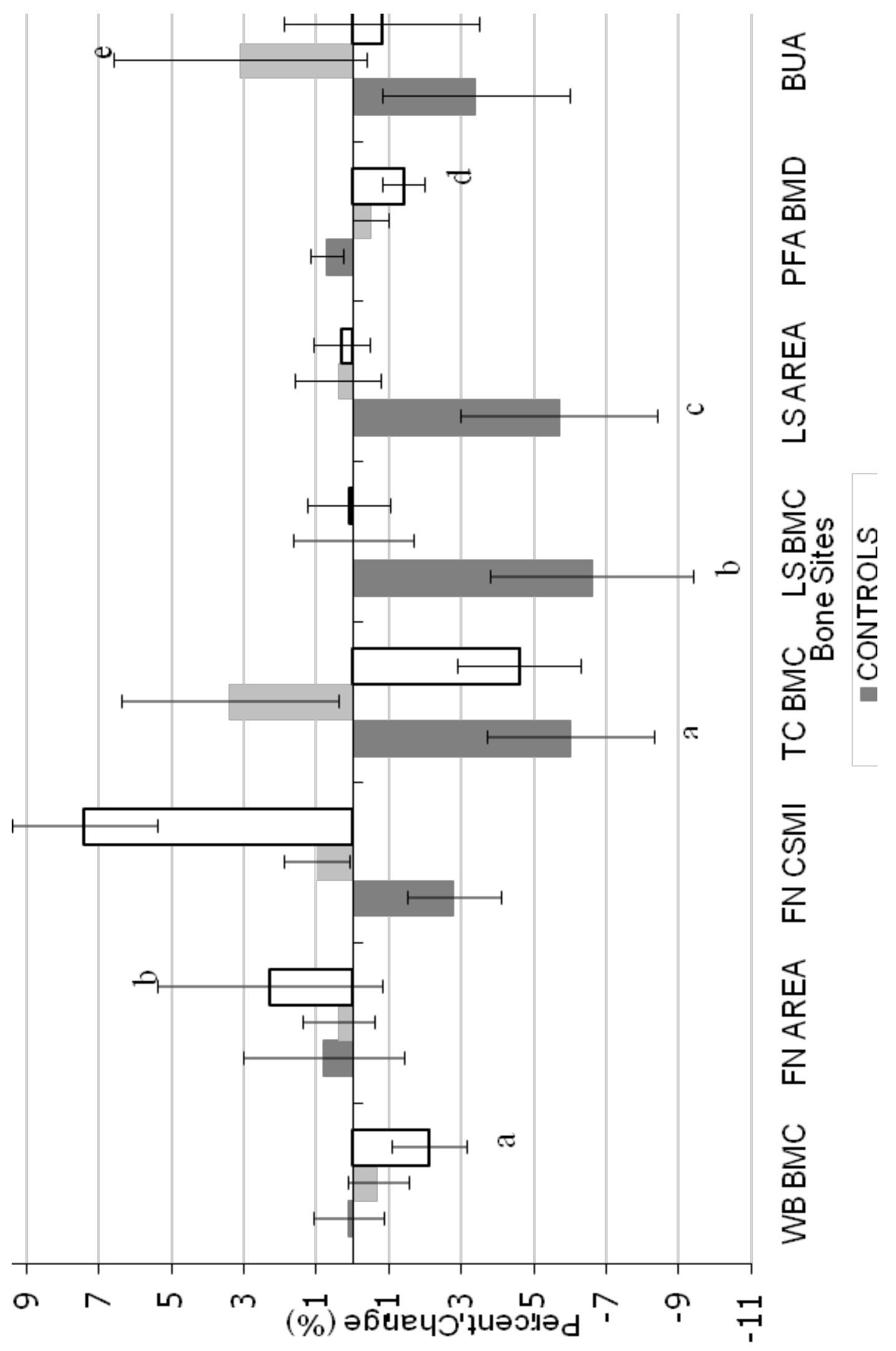




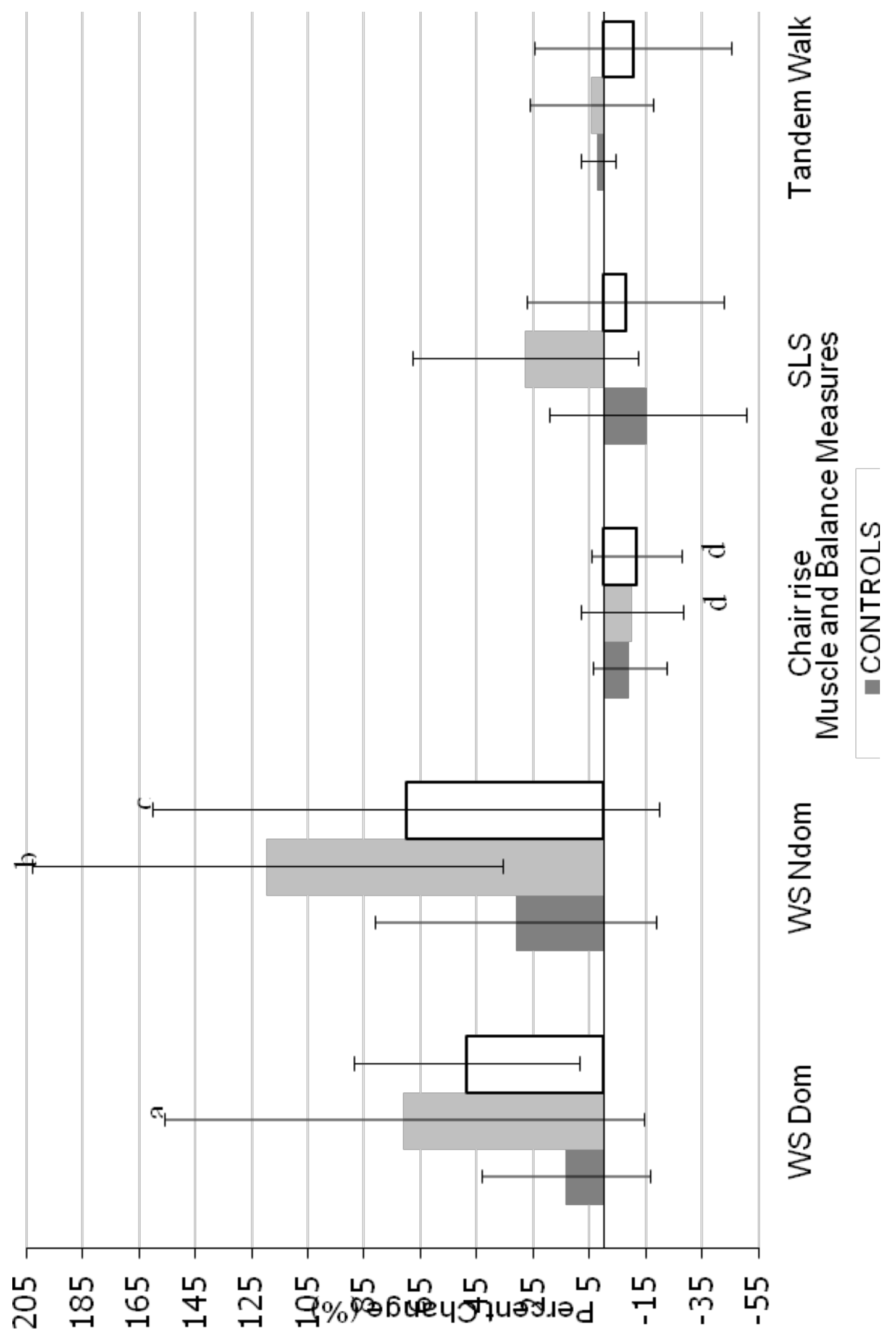


Table 1. Summary of Subject Characteristics at Baseline; Per Protocol Analysis ( $\mathrm{n}=42)$

\begin{tabular}{lccc}
\hline \hline Group & $\begin{array}{c}\text { Control }(\mathrm{N}=14) \\
\text { mean }( \pm \mathrm{s})\end{array}$ & $\begin{array}{c}\text { HiWBV }(\mathrm{N}=15) \\
\operatorname{mean}( \pm \mathrm{s})\end{array}$ & $\begin{array}{c}\text { LoWBV (N=13) } \\
\text { mean }( \pm \mathrm{s})\end{array}$ \\
\hline Age $(\mathrm{y})$ & $74.2(8.1)$ & $68.9(7.0)$ & $68.5(8.6)$ \\
Height $(\mathrm{cm})$ & $159.3(0.06)$ & $157.1(0.06)$ & $160.2(0.07)$ \\
Weight $(\mathrm{kg})$ & $65.1(10.3)$ & $61.4(8.9)^{\mathrm{a}}$ & $68.4(10.3)$ \\
BMI & $25.7(4.2)$ & $24.8(2.9)$ & $26.7(4.4)$ \\
Calcium (mg) & $1135.0(497.5)$ & $1283.0(476.0)$ & $896.3(285.7)$ \\
BPAQ & $2.2(4.8)$ & $4.2(8.1)$ & $7.6(11.3)$ \\
FN BMD T-score & $-2.0(0.9)$ & $-2.1(0.89)$ & $-2.2(1.3)$ \\
\hline
\end{tabular}

${ }^{a}$ significantly less than LoWBV, $\mathrm{p}<0.05$

HiWBV - Higher intensity whole body vibration; LoWBV - low intensity whole body vibration; BPAQ - Bone-specific Physical Activity Questionnaire score; FN - femoral neck; BMD - bone mineral density 
Table 2. Within-Group Change Following 8 months of Twice-Weekly Whole Body Vibration (Per Protocol Analysis, $n=42$ ).

\begin{tabular}{|c|c|c|c|c|c|}
\hline MEASURE & GROUP & $\begin{array}{l}\text { BASELINE } \\
\text { mean }( \pm S E)\end{array}$ & $\begin{array}{c}8 \text { MONTHS } \\
\text { mean }( \pm \text { SE })\end{array}$ & \% CHANGE & P-VALUE \\
\hline \multirow[t]{3}{*}{ Height (cm) } & Control & 159.3(0.06) & $159.3(0.06)$ & 0.0 & 0.89 \\
\hline & HiWBV & $157.1(0.06)$ & $157.2(0.06)$ & 0.1 & 0.54 \\
\hline & LoWBV & $160.2(0.07)$ & $160.6(0.08)$ & 0.2 & 0.08 \\
\hline \multirow[t]{3}{*}{ Weight (kg) } & Control & 65.1(10.3) & $65.6(10.5)$ & 0.8 & 0.78 \\
\hline & HiWBV & $61.4(8.9)$ & $61.5(9.2)$ & 0.2 & 0.14 \\
\hline & LoWBV & $68.4(10.3)$ & $68.4(10.5)$ & 0.0 & 0.92 \\
\hline \multirow[t]{3}{*}{ Percent fat } & Control & $37.2(5.7)$ & $37.2(5.4)$ & 0.0 & 0.67 \\
\hline & HiWBV & $36.6(7.4)$ & $36.9(8.0)$ & 0.8 & 0.23 \\
\hline & LoWBV & $39.2(5.8)$ & $38.8(5.1)$ & -1.0 & 0.76 \\
\hline \multirow[t]{3}{*}{ Lean mass (kg) } & Control & 33.1(4.3) & $33.4(4.1)$ & 0.9 & 0.69 \\
\hline & HiWBV & $31.3(2.9)$ & $31.1(3.5)$ & -0.6 & 0.63 \\
\hline & LoWBV & $33.3(4.2)$ & $34.4(4.0)$ & 3.3 & 0.13 \\
\hline \multirow[t]{3}{*}{ WB BMC (g) } & Control & 2322.4(233.9) & 2323.7(213.7) & 0.1 & 0.80 \\
\hline & HiWBV & 2242.4(330.3) & 2226.3(337.1) & -0.7 & 0.36 \\
\hline & LoWBV & $2402.4(272.3)$ & 2352.2(296.1) & -2.1 & 0.03 \\
\hline \multirow[t]{3}{*}{ FN BMC (g) } & Control & $3.95(0.53)$ & $3.84(0.50)$ & 14.6 & 0.55 \\
\hline & HiWBV & $3.57(0.59)$ & $3.54(0.53)$ & -0.8 & 0.50 \\
\hline & LoWBV & 3.59(1.07) & $3.62(1.07)$ & 0.8 & 0.97 \\
\hline \multirow[t]{3}{*}{ FN Area $(\mathrm{cm} 2)$} & Control & $5.08(0.31)$ & $5.12(0.29)$ & 0.8 & 0.39 \\
\hline & HiWBV & $4.80(0.69)$ & $4.82(0.67)$ & 0.4 & 0.65 \\
\hline & LoWBV & $4.73(0.84)$ & $4.84(0.81)$ & 2.3 & 0.02 \\
\hline \multirow[t]{3}{*}{ FN CSMI } & Control & $3.26(0.97)$ & $3.17(0.51)$ & -2.8 & 0.72 \\
\hline & HiWBV & $2.90(0.59)$ & $2.93(0.63)$ & 1.0 & 0.99 \\
\hline & LoWBV & $3.12(0.58)$ & $3.35(0.67)$ & 7.4 & 0.09 \\
\hline \multirow[t]{3}{*}{ TR BMC (g) } & Control & 8.63(1.86) & $8.11(2.12)$ & -6.0 & 0.03 \\
\hline & HiWBV & 7.37(1.90) & 7.62(1.73) & 3.4 & 0.78 \\
\hline & LoWBV & $7.60(1.70)$ & $7.25(1.90)$ & -4.6 & 0.42 \\
\hline \multirow[t]{3}{*}{ LS BMC (g) } & Control & $40.48(5.60)$ & $37.80(7.51)$ & -6.6 & 0.02 \\
\hline & HiWBV & 37.97(5.54) & $37.97(5.44)$ & 0.0 & 0.96 \\
\hline & LoWBV & $40.67(6.17)$ & $40.70(6.72)$ & 0.1 & 0.76 \\
\hline \multirow[t]{3}{*}{ LS Area (cm2) } & Control & $43.44(4.53)$ & 40.96(6.77) & -5.7 & 0.009 \\
\hline & HiWBV & $43.48(4.31)$ & 43.64(3.83) & 0.4 & 0.72 \\
\hline & LoWBV & 43.89(6.18) & $44.02(5.81)$ & 0.3 & 0.74 \\
\hline \multirow[t]{3}{*}{ PFA BMD (g/cm2) } & Control & $0.579(0.095)$ & $0.583(0.095)$ & 0.7 & 0.27 \\
\hline & HiWBV & $0.551(0.098)$ & $0.548(0.097)$ & -0.5 & 0.15 \\
\hline & LoWBV & $0.582(0.108)$ & $0.574(0.105)$ & -1.4 & 0.005 \\
\hline \multirow[t]{3}{*}{ BUA (dB/MHz) } & Control & 76.18(14.08) & 73.59(11.15) & -3.1 & 0.08 \\
\hline & HiWBV & 68.06(13.17) & 70.16(17.23) & 3.4 & 0.05 \\
\hline & LoWBV & 72.99(20.65) & 72.39(19.98) & -0.8 & 0.44 \\
\hline
\end{tabular}




\begin{tabular}{llcccc}
\hline Wall squat (D) & Control & $43.1(46.0)$ & $48.9(58.7)$ & 13.4 & 0.24 \\
(seconds) & HiWBV & $50.3(43.8)$ & $85.9(60.2)$ & 70.9 & $\mathbf{0 . 0 2}$ \\
& LoWBV & $50.2(37.4)$ & $74.5(64.0)$ & 48.6 & 0.22 \\
Wall squat (ND) & Control & $30.7(40.6)$ & $40.3(56.7)$ & 31.3 & 0.13 \\
(seconds) & HiWBV & $34.8(29.5)$ & $76.4(50.2)$ & 119.5 & $\mathbf{0 . 0 0 4}$ \\
& LoWBV & $45.2(31.2)$ & $76.9(61.4)$ & 70.1 & $\mathbf{0 . 0 3}$ \\
Chair rise & Control & $14.8(1.0)$ & $13.1(1.0)$ & -9.2 & 0.45 \\
(seconds) & HiWBV & $14.9(1.0)$ & $13.4(1.0)$ & -10.1 & $\mathbf{0 . 0 5}$ \\
& LoWBV & $14.3(3.8)$ & $12.8(4.2)$ & -11.5 & $\mathbf{0 . 0 5}$ \\
SLS & Control & $3.2(2.3)$ & $2.7(1.9)$ & -15.6 & 0.56 \\
(seconds) & HiWBV & $3.6(2.1)$ & $4.6(3.8)$ & 27.8 & 0.21 \\
& LoWBV & $3.9(2.2)$ & $3.6(2.8)$ & -7.7 & 0.53 \\
Tandem walk & Control & $30.1(12.5)$ & $30.7(9.8)$ & 2.0 & 0.65 \\
(seconds) & HiWBV & $28.4(9.9)$ & $29.6(9.6)$ & 4.2 & 0.73 \\
& LoWBV & $27.8(10.8)$ & $24.9(6.1)$ & -10.4 & 0.53 \\
\hline
\end{tabular}

HiWBV - higher intensity whole body vibration; LoWBV - low intensity whole body vibration; WB - whole body; FN - femoral neck; TR - trochanter; LS - lumbar spine; PFA proximal forearm; BUA - broadband ultrasound attenuation; BMC - bone mineral content; BMD - bone mineral density; CSMI - cross sectional moment of inertia; D - dominant leg; ND - non-dominant leg; SLS - single leg stance 